\title{
Comments on the optimization of high resolution Fabry-Pérot filtergraphs
}

\author{
G. B. Scharmer
}

Institute for Solar Physics of the Royal Swedish Academy of Sciences, AlbaNova University Center, 10691 Stockholm, Sweden

e-mail: scharmer@astro.su.se

Received 3 March 2005 / Accepted 23 June 2005

\section{ABSTRACT}

In this paper we present results of simulations of Fabry-Pérot (FPI) system performance that address two entirely separate aspects. The first concerns the image quality of FPIs used in telecentric optical setups. We show that the image degradation from phase errors found by von der Lühe \& Kentischer (2000, A\&AS, 146, 499) can largely be compensated by refocusing and that the major cause of image degradation is the pupil apodization discussed by Beckers (1998, A\&AS, 120, 191).

We also discuss the optimization of FPI systems, considering the effects of cavity errors on the homogeneity of the instrument function (transmission profile) across the FOV when two FPIs are combined in a telecentric reimaging system. Our conclusions from several numerical experiments are that telecentric FPI systems that use two or more FPIs with high (94-95\%) reflectivities are likely to show large variations in the instrument profile across the FOV unless the cavity errors are significantly smaller than $2.0 \mathrm{~nm}$. We show, that such homogeneity can be obtained at relatively low "cost" in terms of increased parasitic light levels by lowering the reflectivity of the FPI with the smallest cavity separation rather then lowering the reflectivity of both FPIs. We also demonstrate that the choice of cavity ratios has a strong impact on the homogeneity of the spectral transmission profile across the FOV and that double FPI systems with cavity ratios around 0.3 perform much better than at around 0.6 .

Key words. telescopes - instrumentation: interferometers - instrumentation: polarimeters - techniques: high angular resolution techniques: spectroscopic

\section{Introduction}

During recent years, narrow-band filter systems based on Fabry-Pérot interferometers (FPIs) have been in increasing use at major solar telescopes. Some examples of such double or triple FPI systems are the TESOS (Kentischer et al. 1998a; von der Lühe \& Kentischer 2000; Tritschler et al. 2002), IPM (Cavallini 1998) and IBIS (Cavallini et al. 2003) systems. Such FPI filter systems offer several advantages over classical Lyot type filters. In particular, FPI filter systems allow higher transmission and more rapid tuning. For polarimetric measurements, FPI systems allow the use of a polarizing beam splitter close to the final focal plane, thereby making it possible to strongly reduce cross-talk originating from atmospheric seeing. In contrast, Lyot filters use a linear polarizer as the first element of the filter. This requires any polarizing beam splitter (and other polarization optics) to be located in front of the filter, thus introducing aberration and wavelength differences between the two beams upon passage through the filter and reimaging system. However, FPI systems have a significant disadvantage compared to Lyot filters and other type of filters by requiring reimaging systems that operate at large F-ratios in order to avoid degradation of the performance, as discussed below.
This requirement is a fundamental limitation in the design of FPI systems for large solar telescopes.

The optimization of double and triple FPI filter systems for large future solar telescope was studied in the context of the Large Earthbased Solar Telescope (LEST) (Owner-Petersen 1994), by Darvann \& Owner-Petersen (1994) in a paper that has strongly influenced the design of later FPI systems including TESOS (Kentischer et al. 1998a,b) and the FPI system planned for the Advanced Technology Solar Telescope (ATST) (Keil et al. 2005), as described by Gary \& Balasubramanian (2004), where also alternatives to FPI systems are discussed. The design of these systems have largely focused on designing combinations of FPIs that achieve strong suppression of spectral straylight while allowing reasonably broad pre-filters to be used. In other recent work, emphasis has been on suppresssing ghost images resulting from multiple reflections from the FPI surfaces (e.g. Tritschler et al. 2002; Martinez-Pillet 2004) and on understanding the degradation of image quality, that is in practice unavoidable in any FPI imaging filter system. Beckers (1998) pointed out that telecentric optical setups, for which the FPI etalons are located close to the focal plane and the telescope pupil is collimated, suffer from a wavelength shift across the pupil that leads to a wavelength dependent 
apodisation. This apodization, depending on the half-width of the FPI system and the chosen $f$-ratio, produces a point spread function that may vary strongly with wavelength within the pass-band of the FPI system, thereby degrading the diagnostic usefullness of such data. In a later paper, von der Lühe \& Kentischer (2000) re-investigated the problem and found that optical phase changes, in addition to the apodisation effects studied by Beckers, lead to severe degradation of image quality for telecentric setups. The same authors also pointed out that collimated optical setups, for which the FPIs are located close to the pupil plane, may suffer from severe degradation of image quality due to amplification of polishing and flatness errors by the high reflectivity of the FPI surfaces. Whereas the use of FPIs with collimated optical setups has the undisputable advantage of having a point spread function (PSF) and a spectral transmission function that is the same for every point within the field-of-view, it is therefore not obvious that such setups, used with e.g the IBIS system (Cavallini et al. 2003) also allow high image quality. To our knowledge, the only exhaustive study of such image degradation for FPI systems intended for solar work has been for the IMAX system planned for the SUNRISE ballon platform (Martinez-Pillet 2004).

In this paper we re-investigate the problem of optimizing double and triple FPI imaging systems. We investigate by analytical approximations the amplification of FPI phase errors by the the high reflectivity of the etalons. We demonstrate, by analytical approximations and precise numerical calculations, that the phase errors of FPI systems used with telecentric optical setups can largely be compensated by refocusing and that the major image degrading effect is that of pupil apodization as discussed by Beckers (1998). We also demonstrate that high reflectivities, chosen for strong suppression of spectral straylight in double or triple FPI system, lead to strong spatial variations of the spectral transmission profile when considering achievable cavity errors. Finally, we propose an approach to optimizing double and triple FPI systems that allow excellent image quality as well as a spectral transmission profile that shows small variations, apart from a wavelength shift, across the FOV. These improvements are at the expense of increased spectral straylight.

\section{FPI phase error amplification and image quality}

\subsection{Phase error amplification}

The complex amplitude of of the transmitted electric vector through an FPI is given by e.g Born \& Wolf (1984), and in a more useful form by von der Lühe \& Kentischer (2000) as

$A=\frac{1-R \exp (-\mathrm{i} \Psi)}{1+R^{2}-2 R \cos \Psi}$

where $R$ is the coating (intensity) reflectance, here defined differently from von der Lühe \& Kentischer (2000), and the (double) phase delay between the two plates $\Psi$ is given by

$\Psi=4 \pi n[d+\epsilon] \cos \alpha / \lambda$.

In this equation, $n$ is the refractive index of the FPI cavity, $d$ is the nominal geometrical cavity separation, assumed to have random variations $\epsilon$, and $\alpha$ the angle of the ray with respect to the normal within the cavity. This equation shows that phase delays may occur both because of cavity errors and variations in the angle of incidence. We point out, that the derivation of these equations in principle require the beam to be collimated but that this remains a good approximation for slow converging beams with the etalons located sufficiently far away from the focal plane. For small angles, $\alpha$ is approximately equal to the angle of incidence outside the cavity, $\alpha_{0}$, divided by the refractive index. This allows the usual approximation

$\cos \alpha \approx 1-\alpha_{0}^{2} / 2 n^{2}$.

The phase of the electric vector $\mathrm{A}$ is given by the ratio of the imaginary and real parts of Eq. (1),

$\phi=\arctan \left(\frac{R \sin \Psi}{1-R \cos \Psi}\right)$,

given by von der Lühe \& Kentischer (2000). This equation assumes that the cavity separation is constant across the FPI. When this is not true, a term representing the phase change relative to air should be added. For high-reflectivity etalons, this is a small correction compared to the large phase change from many internal reflections within the FPI that is ignored in the following analysis.

For sufficiently small angles $\alpha_{0}$, we can approximate the phase $\phi$ by its value assuming normal incidence, $\alpha_{0}=0$, and no cavity errors, $\epsilon=0$, plus a correction

$\phi \approx \phi_{0}+\frac{\partial \phi_{0}}{\partial \Psi_{0}} \delta \Psi=\phi_{0}+\delta \phi$

where $\delta \phi$ is the phase error and

$\frac{\partial \phi_{0}}{\partial \Psi_{0}}=\frac{R\left[\cos \Psi_{0}-R\right]}{1+R^{2}-2 R \cos \Psi_{0}}=f\left(\Psi_{0}, R\right)$

$\Psi_{0}=4 \pi n d / \lambda$,

and

$\phi_{0}=\arctan \left[R \sin \Psi_{0} /\left(1-R \cos \Psi_{0}\right)\right]$.

Furthermore,

$\delta \Psi \approx 4 \pi n\left[\epsilon-d \alpha_{0}^{2} / 2 n^{2}\right] / \lambda$.

The phase error amplification function, $f\left(\Psi_{0}, R\right)$, is plotted as a function of wavelength close to the transmission peak at $500 \mathrm{~nm}$ for an airspaced FPI with $d=0.78 \mathrm{~mm}$ and $R=94 \%$. It is evident that this function is strongly peaked at the wavelength of peak transmission, for which $\Psi_{0}$ is an integer multiple of $2 \pi$, but also that $f$ rapidly trails off to small values outside the peak. The function $f$ is quite similar to the intensity transmission profile, shown dashed in Fig. 1, except in the far wings where $f$ reverses sign. At the peak wavelength,

$f_{\text {peak }}=R /(1-R)$.

This shows that any phase errors from cavity errors or oblique rays are multiplied by a factor that is large when the reflectivity of the FPI is high. We expect that the "effective" phase error, suitably averaged over the transmission profile, will be less than the peak value. 


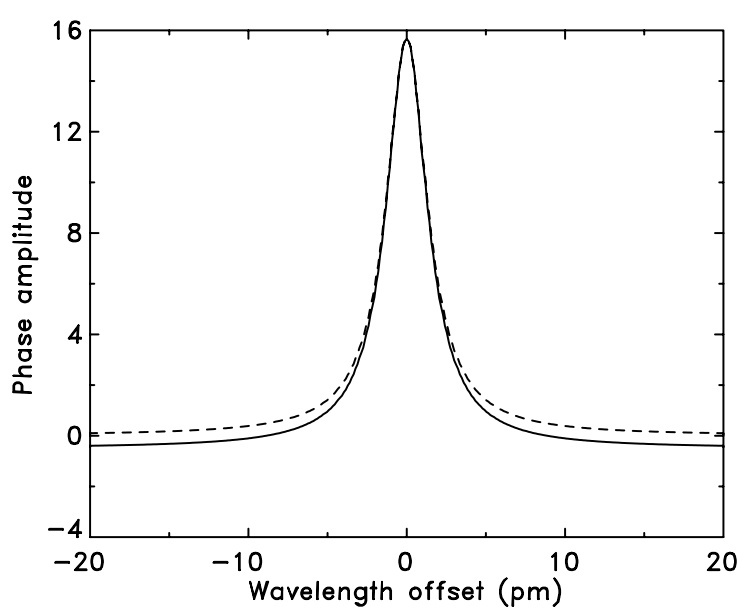

Fig. 1. The phase error amplification function, $f$, where $f$ is defined in Eq. (6) for a single airspaced FPI with cavity separation $0.78 \mathrm{~mm}$ and a reflectivity $R=0.94$ is shown (solid) for a wavelength of $500 \mathrm{~nm}$. Also shown (dashed) is the corresponding intensity transmission profile, normalised to the same peak value as the phase amplitude function.

\subsection{Focus compensation with FPls in telecentric reimaging systems}

In a telecentric setup, the angle of incidence $\alpha_{0}$ varies over the telescope pupil as

$\alpha_{0}=r / 2 F \#$

where $r$ is the pupil radius normalized to unity at the edge and $F \#$ is the F-ratio. When $F \#$ is sufficiently large, the phase error can be written as

$\delta \phi \approx \pi f\left(\Psi_{0}, R\right) d r^{2} /\left(2 n \lambda F \#^{2}\right)$

with a peak value given by

$\delta \phi_{\max }=\pi R d r^{2} / 2(1-R) n \lambda F \#^{2}$.

It is evident from the quadratic variation of the phase error with $r$, that for small angles of incidence, the phase error is in the form of a focus shift that varies in amplitude across the transmission profile. We expect that this phase error can be partly compensated by a simple refocusing of a distance $\delta x$. Such refocusing in air produces a phase error of:

$\delta \phi_{x}=\pi \delta x r^{2} /\left(4 \lambda F \#^{2}\right)$.

Combining Eqs. (13) and (14), we therefore expect that the optimum refocusing distance $\delta x$ should be less than

$\delta x_{\max }=\frac{2 R}{1-R} d / n$.

This approximate equation suggests that for telecentric FPI systems with reflectivities and refractive index that do not vary significantly with wavelength, a simple refocusing should provide image quality improvement over a broad wavelength range. However, small variations in reflectivity may introduce relatively large focusing changes: For $R=0.94$, we obtain $R /(1-R)=15.7$ whereas for $R=0.95, R /(1-R)=19$. Such wavelength variations of the reflectivity by more than $\pm 1 \%$ are typical of high-reflectivity coatings.

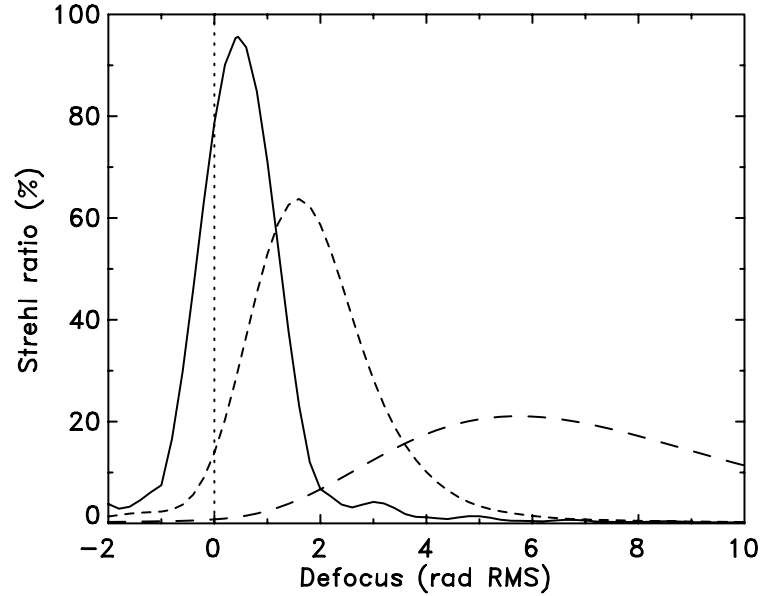

Fig. 2. Strehl ratio as function of defocus parameter $k_{4}$ for $f$-ratios $F \#=256$ (solid), 128 (short dashes) and 64 (long dashes). The Strehl ratios without focus compensation are indicated by the dotted vertical line.

\subsection{Strehl ratio improvement by refocusing}

To investigate the effects of refocusing to compensate phase errors of FPI systems in telecentric setup we have made numerical calculations that include both phase errors and apodization effects and that are not limited to small amplitude phase errors. Re-focusing was simulated by multiplying the complex amplitude $A$ in (1) with a phase term $\exp \left(-\mathrm{i} k_{4} \phi_{4}\right)$ where $\phi_{4}$ is the Zernike polynomial corresponding to focus

$\phi_{4}=\sqrt{3}\left(2 r^{2}-1\right)$,

in units of radians and normalised to 1 radian rms over the pupil, and $k_{4}$ is the corresponding Zernike coefficient. These calculations are valid for arbitrarily large phase errors. We calculated the Strehl ratio for different values of $k_{4}$ and determined the optimum amount of defocus as well as the Strehl ratio with that amount of defocus. For comparison, we also calculated the Strehl ratio without focus compensation and also by zeroing the phase errors, thereby including only the apodization effect discussed by Beckers (1998). We made calculations for a triple air-spaced FPI system similar to those of the TESOS system, with cavity spacings of 1.3, 0.802 and $0.57075 \mathrm{~mm}$ and reflectivity $R=0.94$, and assumed that the complex transmission of the triple system is given by the product of the complex transmissions of the individual FPIs, as was done by von der Lühe \& Kentischer (2000). The calculations were made with 501 wavelengths spanning $\pm 50 \mathrm{pm}$ centered on a wavelength of $500 \mathrm{~nm}$ and with a step size of $0.2 \mathrm{rad}$ for the focusing parameter $k_{4}$. In Fig. 2 are shown the calculated Strehl ratios as function of the focusing parameter $k_{4}$ for $f$-ratios equal to 64,128 and 256 .

It is evident that refocusing gives a dramatic improvement in image quality. The Strehl ratio is increased from $0.8 \%$ $(0.8 \%)$ to $21 \%$ for $F \#=64$, from $14 \%(16 \%)$ to $64 \%$ for $F \#=$ 128 and from $79 \%$ (81\%) to $96 \%$ for $F \#=256$. The Strehl ratios calculated without refocusing are quite similar to the values obtained by von der Lühe \& Kentischer (2000), given within parenthesis above. The corresponding Strehl ratios obtained by zeroing the phase errors, including only the pupil apodization, 
are respectively $28 \%, 71 \%$ and $97 \%$ for $F \#=64,128$ and 256 . It is clear that the degradation of image quality from phase errors is rather small after refocusing and certainly not dramatic, as indicated by the calculations of von der Lühe \& Kentischer (2000). For high Strehl ratios, i.e. small wavefront errors, the focus compensation is nearly perfect.

The amount of focus compensation needed, in terms of wavefront rms, increases with decreasing $f$-ratio. For $F \#=64$, the amount of defocus needed is nearly one wave rms. For these calculations, the defocus needed to obtain optimum Strehl ratio is between $62 \%$ and $77 \%$ of the maximum value calculated from (8) and assuming that each FPI contributes a phase error that scales as $R /(1-R)$. On the other hand, calculations for a single FPI with high reflectivity gave an optimum defocus of only $25-42 \%$ of its expected theoretical maximum value, showing that the theoretical value given by Eq. (12) can only be used to indicate the magnitude of the defocusing needed. This is of no practical importance, since focusing of a real system would be made using a focusing target.

\subsection{Image quality degradation with FPIs in collimated reimaging systems}

One of the most important advantages of using FPI systems with a collimated optical setup and with the FPIs at the pupil plane, is that the spectral transmission profile is the same for every point in the field of view. This is strongly beneficial to the interpretation of sequences of images obtained by scanning narrow spectral lines. In the design of all FPI systems mentioned previously, the effect of cavity errors on the resulting transmission profile has been investigated and accounted for. Ignoring image quality, the effect of cavity errors on the transmission profile is a broadening of the full width at half maximum $(F W H M)$ and a spectral resolution limited by the defect finesse, here defined slightly different from Gary \& Balasubramanian (2004):

$\mathcal{F}_{d}=\lambda /\left(4 n \epsilon_{\mathrm{rms}}\right)$.

Of concern here are the associated effects on image quality. Wavelength shifts from variations in cavity separation are given by

$\delta \lambda_{\mathrm{rms}}=\lambda \epsilon_{\mathrm{rms}} / d$.

The $F W H M$ of the main transmission peak, $\Delta \lambda$, is locally given by the reflectivity as

$\Delta \lambda=\lambda^{2} /\left(2 \mathcal{F}_{\mathrm{r}} n d\right)$

where the reflectivity finesse is

$\mathcal{F}_{r}=\pi \sqrt{R} /(1-R)$.

From these relations, we can calculate the rms wavelength shifts normalized to the FWHM:

$K=\delta \lambda_{\mathrm{rms}} / \Delta \lambda=2 \mathcal{F}_{r} n \epsilon_{\mathrm{rms}} / \lambda=\frac{1}{2} \mathcal{F}_{\mathrm{r}} / \mathcal{F}_{\mathrm{d}}$

When the defect finesse $\mathcal{F}_{\mathrm{d}}$ is comparable to or smaller than the reflectivity finesse $\mathcal{F}_{\mathrm{r}}$, we expect low overall transmission at a fixed wavelength and relatively large transmission variations, in particular at wavelengths corresponding to the wings of the transmission profile. This causes significant apodization and, as explained in the previous sections, phase errors that vary across the FPI plate. When used in collimated setups with the pupil reimaged on the FPI, this will give a degradation of image quality that can be compensated by refocusing only to the extent that the phase error varies quadratically with distance from the center of the etalon. Assuming cavity errors $\epsilon_{\mathrm{rms}}$ of $2 \mathrm{~nm}$, a reflectivity $R=0.94$ and a wavelength of $500 \mathrm{~nm}$, we obtain for an airspaced etalon $\mathcal{F}_{\mathrm{r}}=50.8$ and $\mathcal{F}_{\mathrm{d}}=62.5$. These values are independent of the cavity separation $d$. To estimate the rms phase errors, we assume that the effective wavefront error corresponds to half the peak value. From Eqs. (5)-(10) we obtain

$\phi_{\mathrm{rms}} \approx 2 \pi \frac{R}{1-R} n \epsilon_{\mathrm{rms}} / \lambda$,

valid only for small wavefront errors. An equation similar to Eq. (22) was first derived by von der Lühe \& Kentischer (2000), but based on the peak value of $f$ rather than an estimated $e f$ fective value. These authors alos pointed out the difficulties of achieving high image quality with FPIs located in the pupil plane, even though their actual estimate of the expected wavefront errors appears pessimistic, possibly because of an assumed very high reflectivity. The above equation gives an estimated wavefront rms of 0.4 radians or about $1 / 16$ wave from a single FPI. Thus for cavity errors as small as $2 \mathrm{~nm}$ rms, we expect both significant pupil apodization and significant phase errors when two or more high reflectivity FPIs are used in tandem. The design of FPIs in collimated optical setups for near diffraction limited imaging therefore requires a realistic estimate of cavity errors and a reflectivity chosen to ensure adequate image quality. To our knowledge, the only in-depth analysis of these effects in the design of an FPI system for solar telescopes has been made by Martinez-Pillet (2004) for the IMAX system planned for SUNRISE. By analysing wavefront errors from polished Lithium Niobate $(n \approx 2.3)$ etalons, an upper limit for $R$ that would ensure diffraction limited performance was established.

The use of double or triple FPI systems with high reflectivities at the pupil plane aggravates the problem of pupil apodization and phase errors. In particular, the ratio expressed by $K$ in Eq. (21) is independent of the cavity separation, implying that for a high reflectivity double system, both FPIs contribute to apodization effects. We also emphasize, that the relative wavelength shifts of the peak transmissions for the etalons are larger than the wavelength shifts for the individual FPIs and, according to Eq. (15), dominated by the FPI etalon with the smallest cavity separation.

An additional problem, discussed in the following, is ghost images from multiple reflections at the FPI surfaces. To suppress ghost images from multiple reflections at the FPI surfaces, Tritschler et al. (2002) inserted pre-filter(s) between the FPI(s) for the TESOS system. Such an arrangement, with a tilted pre-filter having peak transmission on the order of $30 \%$, strongly suppresses ghost images by multiple passages of the beam through the prefilter. However, placing the pre-filter(s) 
between the FPIs in a collimated optical setup implies that these filters also need to be of high optical quality to avoid further degradation of image quality.

\section{Performance optimization of FPls in telecentric re-imaging systems}

\subsection{Performance criteria}

In light of the previous discussions, we conclude that telecentric reimaging systems are a safer choice than collimated optical setups as regards high image quality.

A more complicated issue concerns purity of the (spectral) instrument profile. Most future narrowband solar FPI systems are expected to be used also as spectropolarimeters. Such spectropolarimetric data are expected to be analyzed using inversion techniques that build on least squares fit (Skumanich \& Lites 1987), artificial neural networks (Socas-Navarro 2003) or principal component analysis (Socas-Navarro 2001). These techniques use models for the magnetic field, solar atmosphere and for the spectral line observed. From these models, the Stokes vector is calculated at the observed wavelengths and compared to the observed data. The parameters of the models are then adjusted until the agreement between the observed and calculated data is as good as possible according to some chosen norm. For such inversions to produce good results, it is therefore essential that the spectral instrument profile or transmission function of the FPI filter is well known and, in order to reduce complexity of the inversions, constant across the FOV. If this function is well known and the spectral samples sufficiently independent, then small amounts of spectral straylight, hereafter referred to as "parasitic light", are of small importance, provided that this straylight can be modelled and included in the inversions.

In the optimization of solar FPI systems, we therefore propose to give consideration to the following parameters, evaluated with given Strehl ratio and FWHM:

- The parasitic light level, $P$, defined as the integral over wavelength of the transmission profile outside the first minimum of the main transmission peak divided by the integral over wavelength of the transmission profile within the first minimum of the main transmission peak.

- The ghost intensity, $G$, from multiple reflections between the FPIs and calculated as the integral over all wavelengths of the transmitted ghost intensity divided by the integral over all wavelengths of the transmission profile.

- The homogeneity of the center part of the transmission profile over the FOV, here quantified as the rms variation of the $F W H M$ of the transmission profile, $\sigma_{F W H M}$.

- The homogeneity of the wings of the transmission profile over the FOV, here quantified as the rms variation of the asymmetry of the transmission profile.

- The average peak transmission, $T_{\mathrm{p}}$, obtained by calculating the maximum transmission for each individual profile and averaging the result. For two optically perfect and perfectly co-tuned FPIs without absorbtion, reflection losses or alignment errors, $T_{\mathrm{p}}$ is always $100 \%$ irrespective of wavelength shifts and $T_{\mathrm{p}}$ therefore is good indicator of the homogeneity of the transmission profile over the FOV for a telecentric optical system.

The parasitic light level $P$ is very similar to the inverse of the so-called SNR parameter of Darvann \& Owner-Petersen (1994) and also used in the optimization of the TESOS system by Kentischer et al. (1998a).

The ghost intensity $G$ is calculated in a way similar to that indicated in Tritschler et al. (2002) and assuming that the prefilter is located between the two FPIs. If the transmission profiles for the two FPIs is $T_{1}$ and $T_{2}$ and that of the pre-filter is $T_{\mathrm{f}}$ then the overall tranmission is

$T=T_{1} T_{\mathrm{f}} T_{2}$.

Ignoring absorbtion, the intensity of the of the reflected light at the FPIs is $1-T_{1}$ and $1-T_{2}$ respectively (Born \& Wolf 1984) such that the intensity accumulated from the first reflections is

$$
\begin{aligned}
T_{G}= & \left.T\left[\left(1-T_{1}\right)\left(1-T_{2}\right) T_{\mathrm{f}}^{2}\right)\right] \\
& \left.+T\left[\left(1-T_{1}\right)\left(1-T_{2}\right) T_{\mathrm{f}}^{2}\right)\right]^{2} \\
& \left.+T\left[\left(1-T_{1}\right)\left(1-T_{2}\right) T_{\mathrm{f}}^{2}\right)\right]^{3} \\
& +\ldots
\end{aligned}
$$

The total ghost intensity from an infinte number of reflections can then be written as, using that $x+x^{2}+x^{3}+\ldots=x /(1-x)$ (Mack et al. 1963),

$T_{G}=\frac{T\left(1-T_{1}\right)\left(1-T_{2}\right) T_{\mathrm{f}}^{2}}{1-\left(1-T_{1}\right)\left(1-T_{2}\right) T_{\mathrm{f}}^{2}}$.

If the pre-filter is not located between the FPIs, then the ghost intensity is much higher and instead given by

$T_{G}=\frac{T\left(1-T_{1}\right)\left(1-T_{2}\right)}{1-\left(1-T_{1}\right)\left(1-T_{2}\right)}$.

We calculate the quantity $G$ as the integral over all wavelengths of $T_{G}$ divided by the integral over all wavelengths of $T$.

The biggest contributions to the ghost images come from the wings of the transmission profile. For the TESOS dual FPI system, it was noted that the strength of the ghost images varied across the FOV and that the locations of strong ghost images changed when the FPIs were tuned (Kentischer et al. 1998b). To quantify the homogeneity of the ghost images across the FOV, we therefore calculate also the rms of the ghost intensity normalised to its average value.

The asymmetry of the transmission profile is calculated as

$\frac{\int\left(\lambda-\lambda_{0}\right) T(\lambda) \mathrm{d} \lambda}{\int T(\lambda) \mathrm{d} \lambda}$

where $\lambda_{0}$ is the wavelength of peak transmission for each individual transmission profile. This quantity was included to quantify the asymmetry of the wings of the transmission profile, since these are even more sensitive to relative wavelength shifts of the two FPIs than the center of the transmission profile.

Finally, we also calculate the Strehl ratio as described in the previous sections. This allows us to evaluate the image quality degradation resulting from tilting of the FPIs to eliminate ghost images. 


\subsection{Design parameters chosen}

To illustrate the serious effects of cavity errors on the homogeneity of spectral transmission profile and overall performance of FPIs in telecentric re-imaging systems, we investigate a dual FPI system. We have chosen a relatively large $F W H M$ of $3.4 \mathrm{pm}$ at $525 \mathrm{~nm}$ in the absence of cavity errors and for a perfectly collimated beam. In our calculations, we assume a two-cavity $0.3 \mathrm{~nm} F W H M$ pre-filter with a peak transmission of $30 \%$ and located between the FPIs. This system is sufficiently simple that we can discuss and evaluate basic effects without the complications of additional free parameters corresponding to a third FPI and furthermore is similar to the FPI system chosen for the SST.

As was done for the TESOS system, we have made plots of the ratio of the parasitic light level as function of the ratio of cavity separations $d_{2} / d_{1}$, but such that the combined FWHM is fixed at a value close to $3.41 \mathrm{pm}$ at $\lambda=525 \mathrm{~nm}$. In Fig. 3 are shown plots of the parasitic light as function of the cavity ratio $d_{2} / d_{1}$ for a high reflectivity $(R=94 \%)$, high resolution etalon combined with a low resolution FPI with reflectivity of $R=83 \%, R=89 \%$ and $R=94 \%$. Kentischer et al. (1998a) found that when both reflectivities equal $94 \%$, there are two preferred ratios close to e.g. $d_{2} / d_{1} \approx 0.617$ and $d_{2} / d_{1} \approx 0.439$, for which the parasitic light levels are relatively small. For the present system, $d_{2} / d_{1} \approx 0.415$ gives better performance than with $d_{2} / d_{1} \approx 0.439$ and the former value was adopted for our simulations. We also used $d_{2} / d_{1} \approx 0.290$, which is close to the cavity ratio of IBIS and, as an extreme example, also $d_{2} / d_{1}=0.186$ (Rees, private communication). Calculations for cavity ratios $d_{2} / d_{1}=0.234$ and $d_{2} / d_{1}=0.382$ have also been made but are not discussed here. In Fig. 4 are shown plots of the corresponding ghost intensities. The ghost intensities increase monotonically with the cavity ratio and with increasing reflectivity, opposite to what is the case for the parasitic light level. Thus an optimum design depends, among other things, on the choice of prioritizing low parasitic light levels or low ghost intensities.

In our calculations, we target a Strehl ratio in the range 90-95\%.

\subsection{Effects of cavity errors on the spectral transmission profile}

Table 1 summarizes the results of numerical simulations of FPI systems with the four different cavity ratios indicated by "+" symbols in Figs. 3 and 4 and. assuming an F-ratio of 150. For the calculations presented in Table 1, except the calculation of the Strehl intensity and the $F W H M$ at F/150, we added random errors to the cavity separation with gaussian distributions and standard deviations of $2.0 \mathrm{~nm}$. These random errors are used to represent a combination of large-scale flatness and small-scale roughness errors and appear to represent the state-of-the-art of present-day FPIs, unless exceptional efforts are invested. For each of the calculated transmission profiles, we calculated the peak transmission and peak wavelength by quadratic interpolation. We then used linear interpolation to find the wavelengths at $50 \%$ of the peak transmission and used

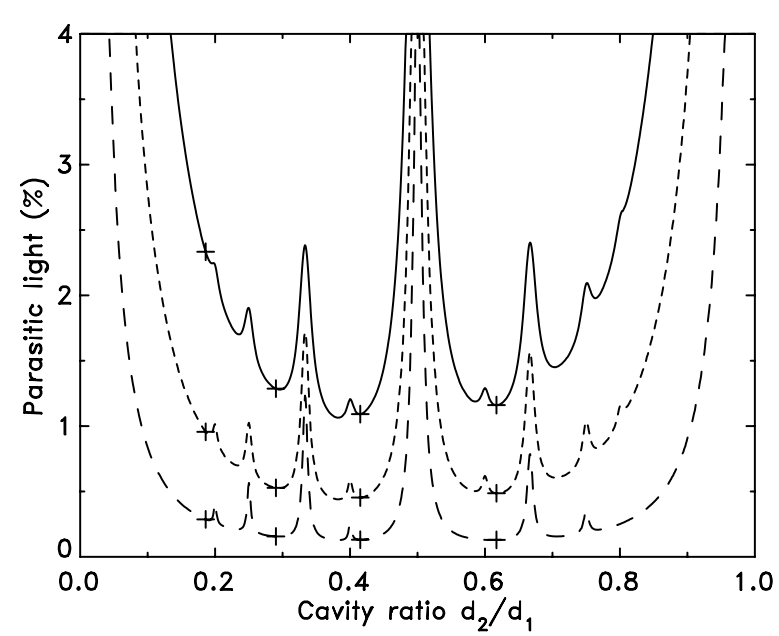

Fig. 3. Parasitic light level in percent as function of the cavity ratio $d_{2} / d_{1}$ but with the cavity separations chosen to give a constant $F W H M$ close to $3.41 \mathrm{~nm}$. Plots are shown for $\lambda=525 \mathrm{~nm}$ with reflectivities of $83 \%$ (solid), $89 \%$ (short dashes) and $94 \%$ (long dashes) for the low resolution FPI. Simulations have been made for the cavity ratios indicated with + symbols and are discussed in the text. The calculations were made for zero cavity errors.

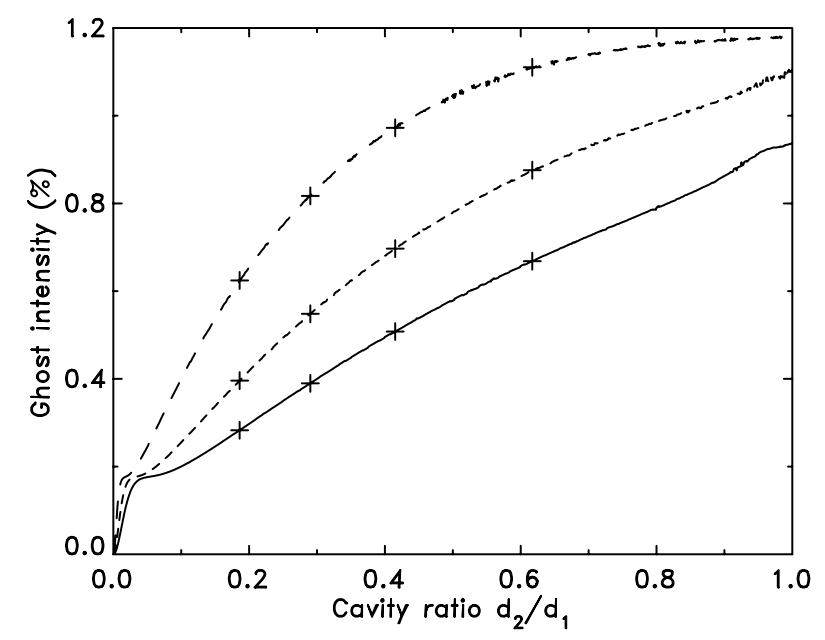

Fig. 4. Ghost intensities in percent as function of the cavity ratio $d_{2} / d_{1}$ but with the cavity separataions chosen to give a constant $F W H M$ close to $3.41 \mathrm{~nm}$. Plots are shown for $\lambda=525 \mathrm{~nm}$ with reflectivities of $83 \%$ (solid), 89\% (short dashes) and 94\% (long dashes) for the low resolution FPI. Simulations have been made for the cavity ratios indicated with + symbols and are discussed in the text. The calculations were made for zero cavity errors.

these wavelengths to calculate the $F W H M$ for each profile. We also calculated the parasitic light level, $P$, and the ghost intensity, $G$, as described previously, for each profile. In Table 1 is given the average $F W H M$ obtained by calculating the $F W H M$ of each individual profile and averaging the calculated widths. Note that this is not the same as calculating the FWHM of the averaged profile. We also give the rms variation of the FWHM. The Strehl intensities and the nominal $F W H M$ at $\mathrm{F} / 150$ but without cavity errors were also calculated for each configuration at $\lambda=525 \mathrm{~nm}$. 
Table 1. Note: All configurations have the same nominal $F W H M$ close to $3.41 \mathrm{pm}$ at $\lambda=525 \mathrm{~nm}$ in the absence of cavity errors and assuming a perfectly collimated beam. Cavity errors of $2.0 \mathrm{~nm}$ rms have been assumed for all calculated parameters except the Strehl ratio and the $F W H M$ at $\mathrm{F} / 150$.

\begin{tabular}{llllllllll}
\hline \hline Parameter & Conf. 1 & Conf. 2 & Conf. 3 & Conf. 4 & Conf. 5 & Conf. 6 & Conf. 7 & Conf. 8 & Conf. 9 \\
\hline Cavity ratio $d_{2} / d_{1}$ & 0.617 & 0.415 & 0.290 & 0.186 & 0.617 & 0.415 & 0.290 & 0.186 & 0.290 \\
Cavity sep. $d_{1}(\mathrm{~mm})$ & 0.6250 & 0.6964 & 0.7371 & 0.7710 & 0.7521 & 0.7760 & 0.7870 & 0.7920 & 1.0749 \\
Cavity sep. $d_{2}(\mathrm{~mm})$ & 0.3856 & 0.2890 & 0.2137 & 0.1433 & 0.4641 & 0.3221 & 0.2281 & 0.1473 & 0.3119 \\
Reflectivity FP1 $(\%)$ & 94 & 94 & 94 & 94 & 94 & 94 & 94 & 94 & 91.4 \\
Reflectivity FP2 $(\%)$ & 94 & 94 & 94 & 94 & 86 & 86 & 86 & 86 & 91.4 \\
\hline Parasitic light $(\%)$ & 0.13 & 0.15 & 0.20 & 0.40 & 0.80 & 0.78 & 0.93 & 1.71 & 0.94 \\
rms Parasitic light $(\%)$ & 34.9 & 38.3 & 41.0 & 45.2 & 12.1 & 12.0 & 12.0 & 12.8 & 12.3 \\
Ghost intensity $(\%)$ & 1.25 & 1.23 & 1.22 & 1.22 & 0.86 & 0.74 & 0.65 & 0.58 & 1.03 \\
rms Ghost intensity $(\%)$ & 26.3 & 34.1 & 43.0 & 54.2 & 18.6 & 28.4 & 39.3 & 53.4 & 38.2 \\
Average $F W H M(\mathrm{pm})$ & 4.34 & 3.90 & 3.69 & 3.53 & 3.50 & 3.45 & 3.43 & 3.42 & 3.60 \\
rms $F W H M(\mathrm{pm})$ & 1.05 & 0.41 & 0.20 & 0.08 & 0.09 & 0.04 & 0.03 & 0.02 & 0.17 \\
rms wavelength shift $(\mathrm{pm})$ & 1.48 & 1.43 & 1.39 & 1.35 & 1.31 & 1.31 & 1.31 & 1.32 & 0.95 \\
rms asymmetry $(\mathrm{pm})$ & 0.76 & 0.89 & 0.98 & 1.06 & 0.41 & 0.43 & 0.45 & 0.45 & 0.62 \\
Transmission at fixed $\lambda(\%)$ & 53.3 & 53.3 & 53.3 & 53.2 & 67.3 & 67.2 & 67.3 & 67.3 & 68.4 \\
Transmission at peak $\lambda(\%)$ & 71.7 & 72.2 & 72.5 & 72.6 & 90.1 & 91.0 & 91.4 & 91.6 & 82.4 \\
Strehl ratio at F/150 $(\%)$ & 93.6 & 94.1 & 94.2 & 94.6 & 94.3 & 94.5 & 94.6 & 94.7 & 94.2 \\
Nom. $F W H M$ at F/150 $(\mathrm{pm})$ & 4.29 & 4.33 & 4.39 & 4.43 & 4.40 & 4.45 & 4.46 & 4.49 & 4.39 \\
\hline
\end{tabular}

We first discuss Configurations 1-4. These calculations were made with reflectivities of $94 \%$ for both etalons. These configurations are characterized by low parasitic light levels, varying with the cavity ratio $d_{2} / d_{1}$ more or less as expected from Fig. 3. The ghost intensities, however, do not follow the curve in Fig. 4 but saturate to a value of around $1.22-1.25 \%$, independent of the cavity ratio. This effect is due to the combination of high reflectivities and (small) random cavity errors. For Configuration 4, these cavity errors result in an increase in ghost intensities by a factor 2 , for Configuration 1 , the increase is only $10 \%$ as compared to assuming zero cavity errors. The rms variations of the ghost intensities are $26-54 \%$ of their average values for Configurations 1-4. Plots of individual ghost profiles show strong intermittency: Most profiles are strongly asymmetric with dominant contributions in either the red or the blue wing. Such asymmetries will give e.g spurious Doppler signals and the more or less erratic variations of the ghost profiles stand out as a major problem.

It is evident that Configurations 1 and 2 cannot produce homogeneous transmission profiles across the FOV. For Configuration 1, the random wavelength shifts of both FPIs produce rms variations in the $F W H M$ that are more than $30 \%$ of the nominal $F W H M$ of $3.41 \mathrm{pm}$. Moreover, as a result of these wavelength shifts, the average $F W H M$ is increased by $27 \%$.

The rms variations of the $F W H M$ decrease strongly with decreasing cavity ratio. These variations are 13 times smaller for Configuration 4 than for Configuration 1. This is because a smaller cavity separation for FPI 2 gives a larger FWHM that can more easily accomodate the relative wavelength shifts between the two FPIs. We emphasize that the relative wavelength shifts between the two FPIs are dominated by the FPI with the smaller cavity separation and that we can consider wavelength shifts primarily from that FPI in combination with its FWHM to give rise to the variations in the transmission profile across the FOV. The asymmetry of the line profile shows a (small) variation with cavity ratio that is opposite to the variation of the rms of the FWHM. This is because for cavity ratios close to 1 , the wings of the transmission profile are much less extended than when $d_{2} / d_{1}$ is small, giving a larger contributions to the asymmetry from wavelengths close to the peak wavelength.

In Configurations 5-8, we have maintained the high reflectivity for the first FP but lowered the reflectivity of the second FPI to $86 \%$. This increases the FWHM of the second FPI by nearly a factor of 2.5 which gives a dramatic improvement in the homogeneity of the transmission profile. The rms variation in the $F W H M$ is reduced by $4-10$ times and the average $F W H M$ is nearly exactly the same as the nominal value for all configurations. The price paid is an increase in the parasitic light level by $5-6$ times while the ghost intensity is actually reduced by $30-50 \%$ and decreases with decreasing cavity ratio, as expected from Fig. 4. As was the case when both reflectivities are high, the rms variations in $F W H M$ decrease strongly with decreasing cavity ratio. We also note that the average peak transmission is $90-92 \%$ for all configurations with $R=86 \%$, suggesting that this parameter can be considered as a quality measure, similar to the Strehl ratio used for imaging systems, for FPIs in telecentric optical setups. For FPIs in collimated optical setups, the corresponding (conventional) quality measure is the transmission at a fixed wavelength. These values are also given in the Table and are in the range 53-68\%. The rms of the ghost intensities remain disturbingly high, in particular for low cavity ratios. In Fig. 5 is shown a randomly chosen 


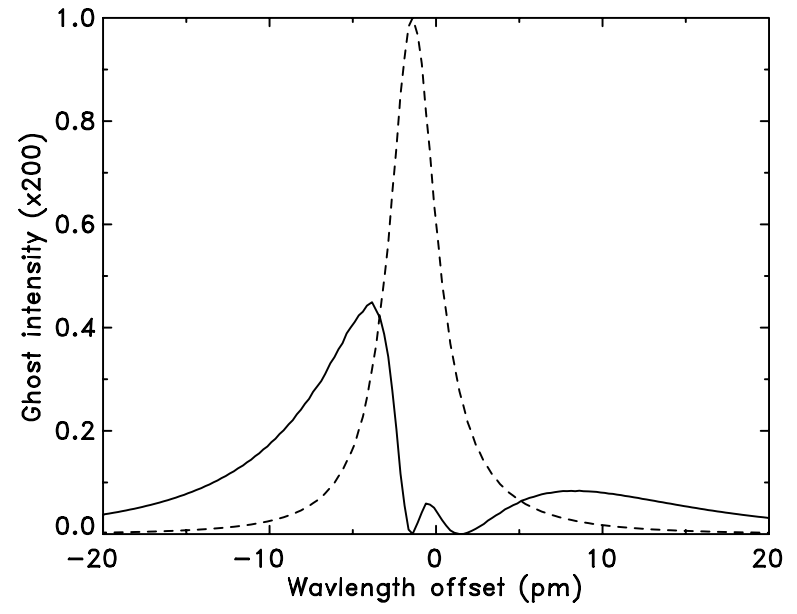

Fig. 5. Randomly chosen example of ghost intensity profile from simulations for Configuration 7. The ghost intensity profile (full) has been amplified 200 times relative to the transmission profile (dashed). The strong asymmetry is evident. The wavelength shifts from cavity errors for the two FPIs can be read off directly from the minima of the ghost profile.

example of a ghost intensity profile for Configuration 7. The strong asymmetry of this profile emphasizes the importance of either suppressing ghost intensities to very low levels or eliminating the ghost images completely. The rms variation of the parasitic light levels are in the range $12-13 \%$ for Configurations 5-8. Reducing the rms cavity errors to $1 \mathrm{~nm}$, corresponding to small scale polishing errors, reduces these variations by a factor four to $3 \%$. We conclude that the parasitic light levels for these configurations should be smooth at small scales and show only small variations over large scales across the FOV.

In a final numerical experiment, we assumed the same reflectivities for both FPIs and varied these reflectivities and the cavity separations, maintaining the ratio of $d_{2} / d_{1}=0.290$ fixed, until the nominal $F W H M$ of $3.41 \mathrm{pm}$ was obtained without cavity errors and the parasitic light level was approximately the same as for Configuration 7. The parameters obtained from these constraints are given for Configuration 9 in Table 1. It is clear from this example, that reducing the reflectivities of both FPIs with the same amount in order to improve the homogeneity of the transmission profile leads to poor performance as compared to lowering the reflectivity of the low resolution FPI. The rms variation in $F W H M$ is nearly six times larger for Configuration 9 than for Configuration 7 and the average FWHM of Configuration 9 is broadened significantly. In addition, the ghost intensity is higher for Configuration 9 than for Configuration 7 . The only benefit of reducing the reflectivities of both FPIs is that the rms wavelength shifts of the transmission peak is reduced by about $30 \%$, due to the increased cavity separations.

The calculated Strehl intensities at F/150 are all in the range of 94-95\% and show only a minor increase with decreasing cavity ratio and decreasing reflectivity of the low resolution FPI. At this $f$-ratio and for $\lambda=525 \mathrm{~nm}$, the broadening of the transmission profile from the converging $\mathrm{F} / 150$ beam increases its $F W H M$ by about $30 \%$.
Of the configurations studied we consider all with $94 \%$ reflectivity for the low-resolution FPI to be unsuitable in a high-fidelity FPI system except possibly Configuration 4 . However, since these calculations were made using very small cavity errors $(2 \mathrm{~nm} \mathrm{rms})$, we strongly prefer the more robust configurations with lower reflectivity for the low resolution FPI (Configurations 5-8). Of these, the performance of Configuration 5 obviously degrades quickly with increasing reflectivity and is therefore rejected. Configuration 8 gives a parasitic light level that is nearly two times higher than for Configurations 6-7 and has no other advantage that compensates for this increase in parasitic light level. Configurations 6 and 7 both indicate good performance in a telecentric optical system, with robustness against variations in reflectivity. We note, that even these "best" systems are compromises: The parasitic light levels and the ghost intensities are on the high side and the transmission profiles show significant and variable asymmetries in the wings that will need calibration.

\subsection{Image quality degradation from tilted FPIs}

In our calculations, we have assumed blocking filters with peak transmissions of $30 \%$. If the peak transmission is higher, the ghost intensities can reach unacceptably high levels. In that case, the FPIs may need to be tilted to completely eliminate the ghost images and the blocking filters can then be moved to a location in front of the FPIs. Such tilt gives rise to image quality degradation, partly due to pupil apodization and partly due to phase errors, that cannot be compensated by refocusing, as well as an asymmetric spectral transmission profile. To investigate the image quality degradation from such tilted FPIs, we have calculated Strehl ratios for various combinations of FPI tilts, all giving a total tilt of $0.19 \mathrm{deg}$ which is needed to allow a pupil stop to eliminate all ghost images with an F-ratio of 150 . Such a pupil stop can be inserted without adding extra optics in the second part of the telecentric system that reimages the FOV onto the CCD. The calculations show that the highest Strehl ratio is obtained with about $15 \%$ and $85 \%$ of this 0.19 deg tilt for the high- and low-resolution FPIs respectively. The Strehl ratio is reduced from $94.6 \%$ to $90.3 \%$ at $525 \mathrm{~nm}$. Increasing the $f$-ratio to $\mathrm{F} / 165$, and a corresponding decrease in the tilt angle, gives a Strehl ratio of $92.9 \%$ which gives an indication of the loss in FOV that would result in recovering the Strehl ratio lost by tilting the FPIs. If indeed we choose to eliminate the ghost images by tilting the FPIs, then we may use a cavity ratio of 0.415 or 0.382 instead of 0.290 , since this gives slightly (about 20\%) reduced parasitic light.

If the peak transmission of the blocking filter is below 50\%, there is also the possibility to keep the blocking filter between the FPIs and to tilt the FPIs by about half the angle given above. This will displace the pupil image of the first reflection by half a pupil diameter and the pupil images of all following reflections by more than a pupil diameter. This reduces the ghost intensity from $0.65 \%$ to $0.25 \%$ if the peak transmission is $30 \%$, from $1.19 \%$ to $0.45 \%$ if the peak transmission is $40 \%$ and from $1.92 \%$ to $0.70 \%$ if the peak transmission is $50 \%$. Such a small tilt angle will have a negligible effect on the Strehl ratio. 


\section{Conclusions}

In this paper we present results of simulations of FPI system performance that address two entirely separate aspects. The first concerns the image quality of FPIs used in telecentric optical setups. We show that the image degradation from phase errors found by von der Lühe \& Kentischer (2000) can largely be compensated by refocusing and that the major cause of image degradation is the pupil apodization discussed by Beckers (1998).

We also discuss the optimization of FPI systems, considering the effects of cavity errors on the homogeneity of the transmission profile across the FOV when two FPIs are combined in telecentric optical setups. Based on numerical calculations, we conclude that telecentric FPI systems that use two or more FPIs with high (94-95\%) reflectivities are likely to show large variations in the transmission profile across the FOV unless the cavity errors are significantly less than $2.0 \mathrm{~nm}$ rms, as assumed for the calculations made. We conjecture that small such variations in FWHM are more important than extremely low levels of parasitic light and propose that future solar FPI systems should make appropriate tradeoffs to ensure such homogeneity. We also show, that such homogeneity can be obtained at relatively low "cost" in terms of increased parasitic light levels by lowering the reflectivity of the FPI with the smallest cavity separation rather then lowering the reflectivity of both FPIs. We also demonstrate that the choice of cavity ratios has a significant impact on the homogeneity of the transmission profile across the FOV and that double FPI systems with cavity ratios around 0.3 or 0.4 perform much better than those with cavity ratios of 0.6 or higher.

As a result of our studies, we are inclined to suggest FPI systems used with telecentric optical setups as a safer solution to achieve high image quality. For such systems, there is no particular penalty in using a high resolution FPI with such high reflectivity that the reflective finesse is larger than the defect finesse, in combination with a low resolution, low reflectivity FPI. Such a combination leads to wavelength shifts that are large compared to the $F W H M$, but the average transmission at the peak wavelength remains very high and the Strehl ratio is only affected by the choice of FWHM and the F-ratio. The center part of the transmission profile will show small variations across the FOV but the wings will show variable asymmetries across the FOV that needs calibration.

For a collimated optical setup with the pupil reimaged on the FPIs, the requirements on roughness and flatness are tighter. For such systems, it is necessary to choose the reflectivity of the high resolution etalon such that the variations in transmission at each fixed wavelength are small across the FPI. This implies that the reflective finesse needs to be significantly smaller than the defect finesse. If this is not the case, the overall transmission may be around or below $50 \%$ and with a risk of significant degradation of image quality from pupil apodization and phase errors. The image quality of an FPI system in collimated optical mount is difficult to estimate, because of the uncertian contribution to flatness errors from stresses in the coatings, which tend to give cavity errors that at least partly can be compensated by refocusing. By restricting the reflectivity to lower values than is possible for a telecentric setup, the parasitic light levels for collimated FPI systems are increased. A complication for FPIs in collimated optical setups is that tilting of the FPIs to eliminate ghost images will introduce relative shifts in the peak wavelength across the FOV. These wavelength shifts will have an effect that is similar to increasing the cavity errors and this will have a negative impact on the overall performance.

For both telecentric and collimated optical setups, we can in principle compensate image degradation effects from the FPI system in postprocessing using e.g. MFBD methods Löfdahl (2002). For telecentric setups, it appears relatively straight forward to model and compensate the image degradation from phase errors and pupil apodization (Löfdahl \& Scharmer in prep.), whereas for collimated optical setups, complicated measurements of phase errors and pupil apodization are needed.

We emphasize that for FPIs in telecentric configuration, the ghost images, originating from multiple reflections between the FPIs, are far out-of focus, the amount of defocus increasing for each reflection, and with major contributions from the wings of the transmission profile, shifted in wavelength by cavity errors. Modelling and compensation of ghost images appear much more difficult than is the case for contributions from parasitic light. This suggests that elimination or suppression of ghost images should be given higher priority than reducing parasitic light levels to very low values. The analysis of Tritschler et al. (2002) indicate that problems from ghost images are significantly worse for triple than for dual FPI systems because of multiple reflections between three instead of two FPIs, the use of pre-filters that allow higher transmission and because triple FPI systems do not allow simple suppression by inserting the blocking filter between two of the FPIs.

In this paper, we have discussed the design of a telecentric dual FPI system similar to that intended for the SST. This design takes into account the effects of cavity errors, at a level close to that of the best FPIs manufactured today. This drives the design towards combining a high resolution, high reflectivity etalon with a low resolution, low reflectivity etalon having a cavity ratio of approximately $0.3-0.4$. We have demonstrated that such a combination gives a reasonably homogeneous (spectral) transmission profile and relatively low ghost levels, at the expense of increased levels of parasitic light. We believe that a double FPI system with one low reflectivity FPI, by reducing the number of reflections within that FPI, also will show relatively low levels of spatial straylight (Rees, private communication). The relatively large $F W H M$ of such a system compared to other existing or planned solar FPI system gives a large FOV while allowing near diffraction limited image quality and rapid scanning of spectral lines without undersampling (aliasing).

It appears likely, that robust triple FPI systems can be designed as an extension of the dual FPI system discussed here but with a third FPI that has lower reflectivity and/or significantly smaller cavity separation than the medium resolution FPI.

Acknowledgements. I want to express my gratitude to Mats Löfdahl for verifying some of the calculated Strehl ratios, 
Valentin Martinez Pillet for verifying early calculations of FPI performance and for numerous discussions, and to Allen Gary, Kai Langhans and Mats Löfdahl for comments on the manuscript.

\section{References}

Beckers, J. M. 1998, A\&AS, 120, 191

Born, M., \& Wolf, E. 1984, Principles of Optics (Pergamon press)

Cavallini, F. 1998, A\&AS, 128, 589

Cavallini, F., Baffa, C., Reardon, K., et al. 2003, Mem. S.A.It., 74, 796

Darvann, A., \& Owner-Petersen, M. 1994, LEST technical report 57, Institute of Theoretical Astrophysics, Oslo

Gary, A., \& Balasubramanian, K. S. 2004, Additional notes concerning the selection of a multiple-etalon system for ATST, Technical note 0027, Advanced Technology Solar Telescope, 950 N. Cherry Avenue, Tucson, AZ, USA, http://atst .nso.edu

Keil, S., J., O., Rimmele, T., et al. 2005, in Proc SPIE, Vol. 5489, Astronomical Telescopes: Ground-based Telescopes

Kentischer, T. J., Schmidt, W., Sigwarth, M., \& Uexküll, M. 1998a, A\&A, 340, 569
Kentischer, T. J., Sigwarth, M., Schmidt, W., \& Uexküll, M. 1998b, TESOS: Telecentric Etalon Solar Spectrometer, Technischer bericht, Kiepenheuer Institut, Freiburg

Löfdahl, M. G. 2002, in Image Reconstruction from Incomplete Data II, ed. P. J. Bones, M. A. Fiddy, \& R. P. Millane, Proc. SPIE, 4792, 146

Löfdahl, M. G., \& Scharmer, G. B. in prep.

Mack, J. E., McNutt, F., Roesler, F. L., \& Chabbal, R. 1963, Appl. Opt., 2, 873

Martinez-Pillet, V. 2004, in Optical, Infrared and Milimeter Space telescopes, ed. J. C. Mather, Proc. SPIE, 5487, 1152

Owner-Petersen, M. 1994, LEST technical report 58, Institute of Theoretical Astrophysics, Oslo

Skumanich, A., \& Lites, B. 1987, ApJ, 322, 473

Socas-Navarro, H. 2001, in Advanced Solar Polarimetry: Theory, Observation and Instrumentation, ed. M. Sigwarth, ASP Conf. Ser., 236, 487

Socas-Navarro, H. 2003, Neural Networks, 16, 355

Tritschler, A., Schmidt, W., Langhans, K., \& Kentischer, T. 2002, Sol. Phys., 211, 17

von der Lühe, O., \& Kentischer, T. J. 2000, A\&AS, 146, 499 